\title{
固态配位聚合物 $\left\{\left[\mathrm{Ag}_{2}(\mathrm{bpp})_{2}\left(\mathrm{H}_{2} \mathrm{O}\right)_{2}\right](\mathrm{chd}) \cdot 9 \mathrm{H}_{2} \mathrm{O}\right\}_{n}$ 中之新颖“三叉星”笼状 $\left(\mathrm{H}_{2} \mathrm{O}\right)_{5}$ 水簇的发现
}

\author{
(1) 华侨大学材料物理化学研究所, 厦门 361021 \\ (2) 华南理工大学化学化工学院, 广州 510640 \\ *通讯作者, E-mail: djc@hqu.edu.cn
}

骆耿耿 ${ }^{(1)}$, 熊红波 ${ }^{(1)}$, 傅志勇 ${ }^{(2)}$, 戴劲草 ${ }^{(1) *}$

收稿日期: 2011-12-12; 接受日期: 2011-12-29; 网络版发表日期: 2012-05-29 doi: $10.1007 / \mathrm{s} 11426-012-4581-2$

摘要固态配位聚合物 $\left\{\left[\mathrm{Ag}_{2}(\mathrm{bpp})_{2}\left(\mathrm{H}_{2} \mathrm{O}\right)_{2}\right](\mathrm{chd}) \cdot 9 \mathrm{H}_{2} \mathrm{O}\right\}_{n}$ (1) (bpp = 1,3-二(4-吡啶基)丙烷、 $\mathrm{H}_{2} \mathrm{chd}$ $=1,4$-环已烷二羧酸)是通过溶液相超声波合成技术获得的, 建立在 $X$ 射线结构分析技术基础上 的结构(单斜晶系 $C 2 / c$ 空间群, $Z=4$; 晶胞参数 $a=30.37$ (2) $\AA, b=9.271(5) \AA, c=18.89(1) \AA ; \beta=$ $\left.128.47^{\circ} ; V=4164(4) \AA^{3}\right)$ 展现出该化合物捕捉了一条有趣的、由“三叉星”笼状五聚体及 $D_{2 h}$ 构型四 聚体水簇交替构成的一维水带, 该化合物之所以新奇在于其代表了被认为是不太稳定的笼状水 五聚体团簇的晶体学范例. 第一性原理的密度涵理论(DFT)计算表明, 笼状五聚体及 $D_{2 h}$ 构型四 聚体水簇的相互连接对于此类欠稳定水簇的稳定性是有益的.

\section{1 引言}

水簇是一类水分子通过氢键集合在一起的水聚 集体, 仔细了解在不同环境中分立状态下的水簇的 大量可能的结构及其稳定性, 有助于人们研究体相 水或冰 ${ }^{[1]}$ 、以及许多生物、化学和物理过程中 ${ }^{[2]}$ 的 水-水相互作用性质. 这类研究已经导致发现了多种 有趣的小水簇 $\left(\mathrm{H}_{2} \mathrm{O}\right)_{n}{ }^{[3]}$ 、以及一维 $(1 \mathrm{D})$ 水簇链 $/{ }^{\text {带 }}{ }^{[4,5]}$ 、 二维 $(2 \mathrm{D})$ 水簇层 ${ }^{[6]}$ 和三维 $(3 \mathrm{D})$ 水簇结构 ${ }^{[7]}$. 在这些小 水簇中, 特别有趣的是在自然界普遍存在的五水聚 集体，如笼合状态的水合物、小分子疏水基团的水化, 以及蛋白质和 DNA 分子表面的水簇等. 然而, 比起 那些更为常见的四核水簇或六核水簇来说, 五水聚 集体的固态结构类型相当有限 ${ }^{[8]}$. 一些理论计算 ${ }^{[9]}$ 预 言, 对于可能存在的五核水簇, 其最稳定的结构形态 应是如下几种类型: “环”、“信封”, 以及“笼”状构型. 因此, 寻找这些小水簇的实验证据及结构模型代表
了一种重大挑战性工作, 这对于拓展我们的化学视 野进入水结构及其特性方面具有十分重要的意义. 具有环状结构的分立五核水簇已为 Liu 等人采用 FIR-VRT 光谱技术所证实 ${ }^{[8 a]}$, 后来得到 $\mathrm{X}$ 射线晶体 学分析技术的进一步确认 ${ }^{[8 d]}$. 但是, 迄今为止高能态 笼状五核水簇仍然是一个缺失的成员，很少发现被 捕获在大多数的主体基质材料中. 为获取此类高能 态笼状五核水簇, 构筑合适的晶质主体从而为稳定 各种水簇拓朴结构提供适宜的环境显得至关重要. 最近，金属有机络合物 ${ }^{[10]}$ 和其他具有强氢键受体或 受体活性位的极性主体化合物, 如四偶氮(tetraaza)、 酚醛分子容器 (phenolic cavitands) $)^{[11]}$ 、含 $\mathrm{N}$ 氧化物 $(\mathrm{N} \text {-oxides) })^{[12]}$ 、咪唑 (imidazoles) ${ }^{[13]}$ 及氨基酸 (amino acids $)^{[14]}$ 已广泛用于稳定水簇, 而具有丰富氢键受体 活性位的有机二羧基类阴离子迄今用于此方面则较 为少见 ${ }^{[15]}$.

另一方面, 近年来无论在溶液化学, 还是在生物 
化学领域, 关于 $\mathrm{X}^{-} \cdot\left(\mathrm{H}_{2} \mathrm{O}\right)_{n}\left(\mathrm{X}=\mathrm{F}^{-}, \mathrm{Cl}^{-}, \mathrm{Br}^{-}, \mathrm{I}^{-}, \mathrm{OH}^{-}, n\right.$ $=1 \sim 6)$ 水簇无机阴离子的结构研究已经成为重要的 话题 ${ }^{[16]}$, 并且由于其适合于作为气溶胶, 以及设计分 子识别研究之 $\mathrm{X}$ 受体简化模型的原因在实验和理论 方面都得到广泛地研究 ${ }^{[17]}$. 但是, 对比前面所提到的 无机 $\mathrm{X}^{-} \cdot\left(\mathrm{H}_{2} \mathrm{O}\right)_{n}$ 体系, 有机二羧基类阴离子水簇结构 迄今仍较少得到关注 ${ }^{[15 a, b]}$. 众所周知, 当羧酸类溶于 水中, 其羧基阴离子通常处于水化离子状态, 而围绕 阴离子水化层的水分子排列肯定是有别于体相水结 构, 因为这是阴离子周围静电场影响极性水分子重 排的结果, 更重要的是, 水化作用强烈地影响了有机 多羧基阴离子的反应性 ${ }^{[18]}$.

我们最近在研究诱导水簇自组装的有机多羧基 阴离子类模板效应时, 获得了一例捕获了一种新颖 水簇客体的全新银配位聚合物- $-\left[\mathrm{Ag}_{2}(\mathrm{bpp})_{2}\left(\mathrm{H}_{2} \mathrm{O}\right)_{2}\right]$ (chd) $\left.\cdot 9 \mathrm{H}_{2} \mathrm{O}\right\}_{n}$ (1) (式中 bpp = 1,3-二(4-吡啶基)丙烷、 $\mathrm{H}_{2} \mathrm{chd}=1,4$-环己烷二羧酸). 本文在此报道该化合物 的合成、结构, 及其表征. 这个固态聚合物呈现一种 含有银-bpp 阳离子层状主体与水-chd 阴离子层状客 体的超分子三文治式结构, 其中, 前一主体层是由银 与 $\mathrm{bpp}$ 配体组成的聚合链通过 $\mathrm{Ag} \cdots \pi$ 相互作用交替构 成, 而后一客体层则由未配位的 $\mathrm{chd}^{2-}$ 客体阴离子模 板环绕水聚集体构成. 特别有趣的是可观察到一条 新奇的、由罕见的“三叉星”笼式五水聚体与 $D_{2 h}$ 四水 聚体交替构筑而成的一维水带出现在这一氢键连接 的阴离子层中. 这是一例五水聚体笼式构型的晶体 学新颖范例, 其水簇的稳定化能也同时通过采用密 度乏涵理论(DFT)计算得到了评估.

\section{2 实验部分}

\section{1 材料与方法}

合成实验所用到的所有试剂与溶剂均购自商业 试剂公司, 没有进一步纯化; $\mathrm{C} 、 \mathrm{H}$ 和 $\mathrm{N}$ 元素微分析 是在 $\mathrm{CE}$ 仪器公司的 EA 1110 分析仪上完成; 样品的 FT-IR 光谱采用 $\mathrm{KBr}$ 压片法在 4000 400 $\mathrm{cm}^{-1}$ 范围用 Nicolet Nexus 470 红外光谱仪收录; 25 800 ${ }^{\circ} \mathrm{C}$ 范围 的 TGA 热重曲线由 TA5200/MDSC2910 型仪器在 $\mathrm{N}_{2}$ 保护, 加热速率为 $5{ }^{\circ} \mathrm{C} / \mathrm{min}$ 条件下完成测试; 采用 Bruker 公司的 AXS D8 衍射仪、 $\mathrm{Cu}$ 靶在 $40 \mathrm{kV} 、 40$ $\mathrm{mA} 、 2 \theta$ 扫描速率为 $5 \%$ min、步长 $0.1^{\circ}$ 测试条件下收 录磨细样品在 $2 \theta$ 范围为 $5^{\circ} \sim 50^{\circ}$ 的粉末 $\mathrm{X}$ 射线衍射
(PXRD)图; 样品的荧光发射谱是在 Edinburgh 公司的 FL-FS920 TCSPC 型荧光仪上完成测试的.

\section{2 化合物 $\left\{\left[\mathrm{Ag}_{2}(\mathrm{bpp})_{2}\left(\mathrm{H}_{2} \mathrm{O}\right)_{2}\right](\mathrm{chd}) \cdot 9 \mathrm{H}_{2} \mathrm{O}\right\}_{n}(1)$ 的 合成}

把 $\mathrm{Ag}_{2} \mathrm{O}(115 \mathrm{mg}, 0.5 \mathrm{mmol}) 、 \mathrm{bpp}(200 \mathrm{mg}, 1 \mathrm{mmol})$ 和 $\mathrm{H}_{2} \mathrm{chd}\left(174 \mathrm{mg}, 1 \mathrm{mmol}\right.$ ) 的混合物置于 $\mathrm{CH}_{3} \mathrm{OH}-\mathrm{H}_{2} \mathrm{O}$ 混合溶剂(10 mL, $V / V: 1: 4)$ 中, 在室温下采用超声波 $\left(160 \mathrm{~W}, 40 \mathrm{kHz}\right.$ ) 处理 $10 \mathrm{~min}$, 然后, 再用 $\mathrm{NH}_{3}$ 水溶 液 $(25 \%)$ 滴加到上述混合物中至溶液澄清为止. 把这 些澄清液置于室温黑暗环境中缓慢蒸发, 几天后即 获得化合物 1 的无色晶体(基于 $\mathrm{Ag}_{2} \mathrm{O}$ 的收率为 43\%), 在其他 $\mathrm{Ag}_{2} \mathrm{O}: \mathrm{bpp}: \mathrm{H}_{2}$ chd 化学计量比条件下也可制得 化合物 1. 产物经少量的冷 $\mathrm{CH}_{3} \mathrm{OH}$ 和乙醚洗涤. 元素 分析 $\left(\mathrm{C}_{34} \mathrm{H}_{60} \mathrm{Ag}_{2} \mathrm{~N}_{4} \mathrm{O}_{15}\right)$ 理论计算结果(\%)为: C 41.64、 $\mathrm{H}$ 6.17、 N 5.71, 实际测试结果为: C 41.28、H 6.09、 $\mathrm{N}$ 5.83. FT-IR $\left(\mathrm{KBr}, \mathrm{cm}^{-1}\right)$ 光谱: $v=3420(\mathrm{~s}) 、 2950(\mathrm{~m})$ 、 2864(w)、1611(s)、1557(s)、1461(w)、1425(m)、1293(m)、 1256(w)、1221(m)、1009(w)、925(w)、810(m)、756(w)、 611(m)、570(w)、515(m) 和 466(w).

\section{3 单晶结构测定}

在光学显微镜下, 选择具有合适尺寸大小的化 合物 1 单晶, 迅速包覆 Dow Corning 公司出产的真空 脂, 再粘结到玻璃丝上, 并装上载晶台用于衍射数据 收集; 利用装有 $\mathrm{Mo}$ 靶 $(\lambda=0.71073 \AA)$ 、石墨单色器的 日本理学 R-AXIS RAPID IP 单晶衍射仪在 $50 \mathrm{kV}$ 及 $90 \mathrm{~mA}$ 运行条件下采用 $\omega$ 扫描模式测试收集化合物 $\mathbf{1}$ 衍射数据, 总共收录了 $44 \times 5.00^{\circ}$ 组衍射画面, 每组 暴光时间为 $5.0 \mathrm{~min}$. 采用 $\mathrm{ABSCOR}$ 软件 ${ }^{[19]}$ 对数据进 行对称等效反射吸收校正, 选择了最为可能的空间 群, 运用 SHELXS-97 $7^{[20]}$ 软件采用直接法完成结构解 析, 通过 SHELXL-97 ${ }^{[21]}$ 软件采用 $F^{2}$ 全矩阵最小二乘 法对结构进行精修. 非氢原子位置是直接从差分 $F$ 图中找出, 然后用最小二乘法精修确定, 有机分子上 的氢原子坐标采用理论加氢方法按其各向同性温度 因子 1.2 1.5 倍于碳原子的 $U_{\mathrm{eq}}$ 值来确定, 而水分子上 的氢原子位置则直接从差分 $F$-图中求出, 并精修至 $\mathrm{O}-\mathrm{H}=0.85 \AA$ 及 $U_{\text {iso }}(\mathrm{H})=1.2 U_{\mathrm{eq}}(\mathrm{O})$. 经 $\operatorname{PLATON}^{[22]}$ 软件的 Addsym 子程序检验所解析结构, 确认已无额 外对称性. 晶体结构图采用 Diamond v3.1. ${ }^{[23]}$ 软件绘 制. 模拟的 PXRD 图谱采用 Material Studio 软件 ${ }^{[24]}$ 
在单晶数据基础上拟合而成, 化合物 $\mathbf{1}$ 的部分晶体学 数据归纳在表 1 .

\section{3 结果与讨论}

\section{1 合成}

为避免光分解, 化合物 $\mathbf{1}$ 的合成是在黑暗避光条 件下进行的, 详见实验部分的描述. 众所周知, 在水 溶液中 $\operatorname{Ag}(\mathrm{I})$ 与多羧酸根类以及中性配体的反应通常 会产生微晶或无定形银盐沉淀, 这大概是由于银离 子与羧基的快速配位导致形成聚合物结构所致 ${ }^{[25]}$, 因而难以生长质量较好的配合物单晶. 适当地减慢 反应速度或是增加反应物溶解性, 例如采用氨化技 术、层离扩散、凝胶渗透法, 或甚至采用微波辅助水 热法等, 可以用来获得较好的晶质产物 ${ }^{[26]}$. 本工作成 功地采用氨化条件下通过超声波处理的合成策略来 解决上述沉淀难题, 在大约 1 周左右可以获得满足结 构测试的优质单晶化合物 $\mathbf{1}$, 并具良好的合成重复性, 其制备合成对配体与金属之化学配比不太敏感, 表 明也许超声波处理及氨化条件比反应物化学计量比 例更重要. 晶体产物可稳定存于空气环境中, 且不溶 于水和其他有机溶剂. 化合物 $\mathbf{1}$ 分子式 $\left\{\left[\mathrm{Ag}_{2}(\mathrm{bpp})_{2}\right.\right.$ $\left.\left.\left(\mathrm{H}_{2} \mathrm{O}\right)_{2}\right](\mathrm{chd}) \cdot 9 \mathrm{H}_{2} \mathrm{O}\right\}_{n}$ 首先出自低温单晶 $\mathrm{X}$ 射线衍射

表 1 化合物 1 的晶体学及结构精修数据

\begin{tabular}{cc}
\hline Complex & $\mathbf{1}(\mathrm{CCDC}-812566)$ \\
\hline Empirical formula & $\mathrm{C}_{34} \mathrm{H}_{60} \mathrm{Ag}_{2} \mathrm{~N}_{4} \mathrm{O}_{15}$ \\
Formula weight & 980.60 \\
Crystal system & monoclinic \\
Space group & $C 2 / \mathrm{c}$ \\
$a(\AA)$ & $30.37(2)$ \\
$b(\AA)$ & $9.271(5)$ \\
$c(\AA)$ & $18.89(1)$ \\
$\beta\left({ }^{\circ}\right)$ & 128.47 \\
$Z, D_{\text {calc }}\left(\mathrm{mg} \mathrm{m}^{-3}\right)$ & $4,1.564$ \\
$V\left(\AA^{3}\right)$ & $4164(4)$ \\
$\mu\left(\mathrm{mm}^{-1}\right)$ & 1.05 \\
$F(000)$ & 2024 \\
Total No. of reflns. & 7899 \\
No. of unique reflns. & $4068\left(R_{\text {int }}=0.0356\right)$ \\
No. of variables & 3782 \\
Parameters & 249 \\
Final $R$ indices $[I>2 \sigma(I)]$ & $R_{1}=0.0443, w R_{2}=0.1066$ \\
$R$ indices (all data) & $R_{1}=0.0481, w R_{2}=0.1092$ \\
GOF & 1.065 \\
\hline
\end{tabular}

a) $R_{1}=\Sigma|| F_{\mathrm{o}}|-| F_{\mathrm{c}}|/ \Sigma| F_{\mathrm{o}} \mid$; b) $\left.w R_{2}=\left[\Sigma w\left(F_{\mathrm{o}}{ }^{2}-F_{\mathrm{c}}{ }^{2}\right)^{2}\right] / \Sigma w\left(F_{\mathrm{o}}{ }^{2}\right)^{2}\right]^{1 / 2}$.
晶体学的确认, 后经元素分析、IR 光谱及热重分析得 以进一步证实.

\section{2 结构}

图 1 示出了化合物 $\mathbf{1}$ 结构单元的 ORTEP 图, 其 选择性键长和键角列于表 2. 单晶 $\mathrm{X}$ 射线衍射揭示了 化合物 1 由一维无限正弦曲线链式的银-bpp 阳离子 配位聚合物主体和 $\mathrm{chd}^{2-}$ 及水簇阴离子客体构成, 其 不对称单元包含一个银原子、一个 bpp 配体、半个 $\mathrm{chd}^{2-}$ 阴离子、一个水配体, 以及五个结晶水分子. 在 主体部分, 每个 $\mathrm{Ag}$ 中心为三配位的 $\mathrm{T}$ 型配位点, 键 联了两个来自于不同 $\mathrm{bpp}$ 配体的氮原子 $\mathrm{N} 1$ 和 $\mathrm{N} 2{ }^{\mathrm{i}}\left(\mathrm{Ag} 1-\mathrm{N} 1=2.140(3) \AA\right.$ 及 $\left.\mathrm{Ag} 1-\mathrm{N} 2^{\mathrm{i}}=2.138(3) \AA\right)$ 以 及一个弱配位水分子 $\mathrm{O} 1 \mathrm{w}(\mathrm{Ag} 1-\mathrm{O} 1 \mathrm{w}=2.636(3) \AA)$. 通常, 柔性 bpp 配体分子会有四种不同的构象、限定 了不同的 $\mathrm{N} \cdots \mathrm{N}$ 距离(分别为 $T T$ 9.1 10.1 $\AA$ 、 $T G$ 8.6 $9.2 \AA 、 G G^{\prime} 6.7 \sim 8.6 \AA$, 及 $G G 3.9 \AA$, 此处 $T=$ Trans、 $G=$ Gauche $)^{[27]}$. 而在当前化合物中, 可以发现每个 bpp 配体采取了 $T T$ 构象 $(\mathrm{N} \cdots \mathrm{N} 9.17 \AA$ 形式, 且作为一 种 $\mu_{2}$-双啮分子桥联到两个银原子上, 导致产生了一 条具有重复单元为 $[\mathrm{Ag}(\mathrm{bpp})]$ (周期为 $23.775 \AA$ ) 的一 维正弦曲线阳离子链, 所有配位水分子均位于该链 的一侧, 几乎与吡啶环平行 (图 S1(a), 支持性材料). 而且, 相邻的一维阳离子链以反平行方式通过 bpp 配 体最为靠近的芳环与银中心之间存在有效的 $\mathrm{Ag} \cdots \pi$ 相互作用, 其中从芳环碳到 $\mathrm{Ag}(\mathrm{I})$ 中心最短距离 (3.252 ̊) 处于 $A g-\eta^{6}$ 芳烃 $\pi$ 相互作用 2.89 3.37 ̊范

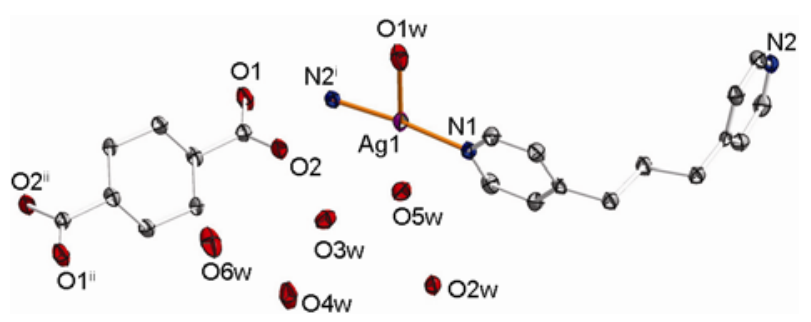

图 1 化合物 1 之晶体结构单元的 ORTEP 图(35\%椭球热振 比率), 为清晰起见, 省略所有氢原子; 对称码: (i) $x+1 / 2$, $-y+1 / 2, z+1 / 2$; (ii) $-x+1 / 2,-y+3 / 2,-z+1$

表 2 化合物 1 的选择性键长 $(\AA)$ 和键角 $\left(^{\circ}\right)$

\begin{tabular}{cccc}
\hline Ag1-N1 & $2.140(3)$ & Ag1-N2 & $2.138(3)$ \\
Ag1-O1w & $2.636(3)$ & N2 ${ }^{\mathrm{i}}-\mathrm{Ag} 1-\mathrm{N} 1$ & $171.7(1)$ \\
$\mathrm{N} 2^{\mathrm{i}}-\mathrm{Ag} 1-\mathrm{O} 1 \mathrm{w}$ & $92.3(1)$ & $\mathrm{N} 1-\mathrm{Ag} 1-\mathrm{O} 1 \mathrm{w}$ & $96.0(1)$ \\
\hline
\end{tabular}

a) Symmetry codes: (i) $x+1 / 2,-y+1 / 2, z+1 / 2$ 
围 ${ }^{[28]}$ 的上限, 促使这些阳离子链进一步排列成一种 二维的超分子层片 $\left\{\left[\mathrm{Ag}_{2}(\mathrm{bpp})_{2}\left(\mathrm{H}_{2} \mathrm{O}\right)_{2}\right]^{2+}\right\}_{n}$ (图 $\left.\mathrm{S} 1(\mathrm{~b})\right)$, 结果导致配位水分子错列在层片两侧. 正如后面会 讨论的，这种错列水分子参与了客体氢键层的构筑， 因而对稳定水簇客体起了重要作用. 涉及 $\operatorname{Ag}(\mathrm{I})$ 中心 的键长和键角均相当于那些观察到的几例同类化合 物 ${ }^{[29]}$.

在客体部分, $\mathrm{H}_{2} \mathrm{chd}$ 分子完全脱除质子成了 $\mathrm{chd}^{2-}$ 阴离子, 作为一种结构导向剂及抗衡阴离子平衡了 聚合物主体的电荷, 而并没有参与 $\operatorname{Ag}(\mathrm{I})$ 中心的配位, 这反映了 $\mathrm{Ag}(\mathrm{I})$ 原子对 $\mathrm{bpp}$ 配体的配位作用强于 $\mathrm{H}_{2} \mathrm{chd}$ 分子. $\mathrm{chd}^{2-}$ 阴离子的两性分子行为诱导结晶水 分子聚集成一种有序的、具有新颖氢键网络系统的二 维水-chd 阴离子层 $\left\{\left[(\mathrm{chd}) \cdot 9 \mathrm{H}_{2} \mathrm{O}\right]^{2-}\right\}_{n}$ (图 2, 上). 沿着 大致 [010]方向, 可观察到该客体层平行于上述主体 $\left\{\left[\mathrm{Ag}_{2}(\mathrm{bpp})_{2}\left(\mathrm{H}_{2} \mathrm{O}\right)_{2}\right]^{2+}\right\}_{n}$ 层片. 有趣的是每个 $\mathrm{chd}^{2-}$ 阴

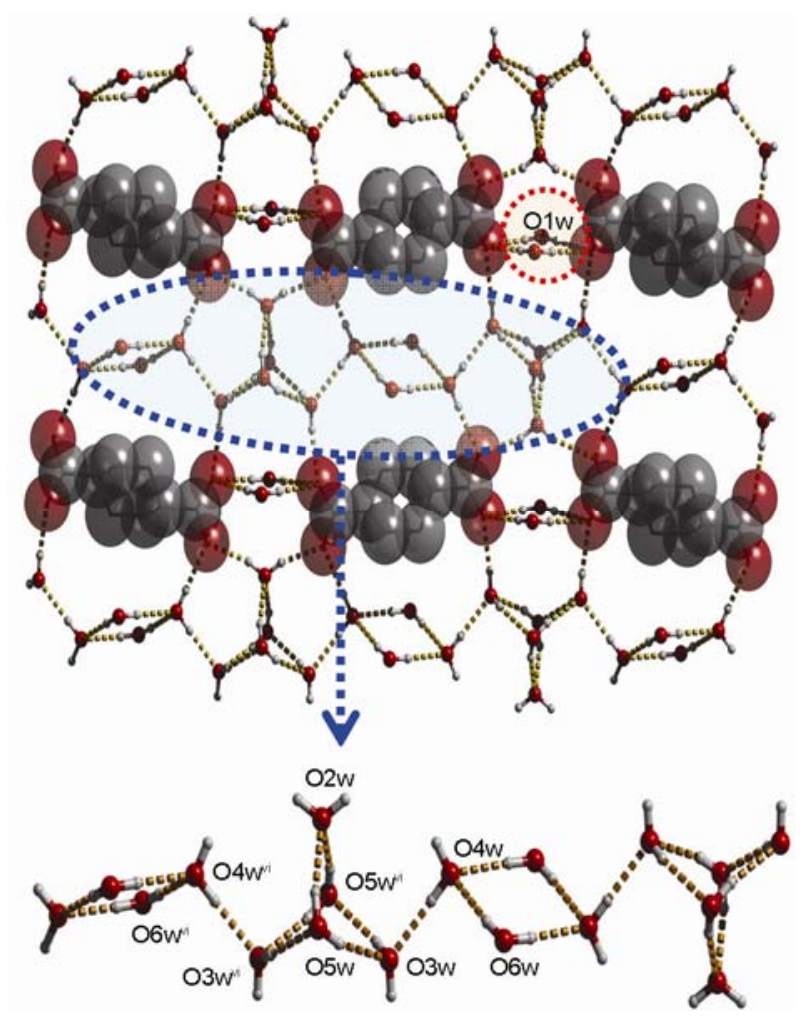

图 2 由 $\mathrm{chd}^{2-}$ 阴离子及结晶水分子组成的氢键网络组装而 成的一种有趣的 $\left\{\left[(\mathrm{chd}) \cdot 9 \mathrm{H}_{2} \mathrm{O}\right]^{2-}\right\}_{n}$ 层(上图), 该结晶水分子 进一步聚集成一条由新奇的“三叉星”笼式五核水簇与 $D_{2 h}$ 四 核水簇交替构筑的一维水带(下图). 注意同时也是聚合物主 体成员之 $\mathrm{O} 1 \mathrm{w}$ 水分子的位置及氢键效应, 显然它对于整个 客体阴离子层的稳定性至关重要. 对称码: (vi) $-x, y,-z+1 / 2$
离子的环已烷骨架的两侧各伴绕着五个水分子, 从 而形成了阴离子簇 $\mathrm{chd}^{2-} \cdot\left(\mathrm{H}_{2} \mathrm{O}\right)_{10}$. 沿着碳骨架中线, 两个等价的水化壳几乎均匀地分布在该阴离子簇两 侧. 其中, 其 $\mathrm{chd}^{2-}$ 的羧基作为氢键受体, 而氢键给体 则由结晶水担任. 环绕 $\mathrm{chd}^{2-}$ 阴离子的水化壳并不同 处一平面, 无疑这是不均匀的环已烷骨架择形诱导 造成的，这与同类的对苯二羧基较平坦的芳环模板 效应诱导是相当不同的 ${ }^{[15 a]}$. 据我们所知, 二羧酸微 溶剂化作用及其在水相中之行为对于大气化学十分 重要 ${ }^{[30]}$. 然而, 大多数关于分立型二羧酸-水簇的报 道仍然主要停留在理论计算模型上 ${ }^{[18]}$, 因此当前的 $\mathrm{chd}^{2-} \cdot\left(\mathrm{H}_{2} \mathrm{O}\right)_{10}$ 水簇阴离子在晶体学水准上的水化壳层 可为人们了解二羧酸类与水相之间的相互作用提供 重要的知识见解.

如图 2(上)所示, 在 $\left\{\left[(\mathrm{chd}) \cdot 9 \mathrm{H}_{2} \mathrm{O}\right]^{2-}\right\}_{n}$ 阴离子层, 靠得最近的 $\mathrm{chd}^{2-}$. $\left(\mathrm{H}_{2} \mathrm{O}\right)_{10}$ 簇由水分子 $\mathrm{O} 1 \mathrm{w}$ 和 $\mathrm{O} 2 \mathrm{w}$ 桥 联在一起(这个 $\mathrm{O} 1 \mathrm{w}$ 是聚合物主体的一个配位成员, 所以它对稳定整个阴离子客体层显得尤其重要), 沿 着大致的 [101]方向产生了“头-尾相联”式的 chd-水 带, 并与相邻水带通过氢键作用进一步缩合成当前 的二维阴离子层片. 图 2 给出了在 $\mathrm{chd}^{2-}$ 阴离子及水 分子之氢键作用范围内结晶水分子的连接关系图示, 所有氢键参数罗列在表 3. 正如我们所见的, 由小五 聚体 $\left(\mathrm{H}_{2} \mathrm{O}\right)_{5}$ 与四聚体 $\left(\mathrm{H}_{2} \mathrm{O}\right)_{4}$ 团簇交替而成的一条一 维新奇水带出现了, 可以发现有趣的双雉式水五聚 体是由三个水分子 $(\mathrm{O} 2 \mathrm{w} 、 \mathrm{O} 3 \mathrm{w}$ 和 $\mathrm{O} 5 \mathrm{w})$ 及其两个等价 对称的水分子 $\left(\mathrm{O} 3 \mathrm{w}^{\mathrm{vi}}\right.$ 、 $\left.\mathrm{O} 5 \mathrm{w}^{\mathrm{vi}}\right)$ 通过氢键作用构筑而 成，其中 $\mathrm{O} 2 \mathrm{w} 、 \mathrm{O} 5 \mathrm{w}$ 和 $\mathrm{O} 5 \mathrm{w}^{\mathrm{vi}}$ 水分子处于对称面上， 而 $\mathrm{O} 3 \mathrm{w}$ 和 $\mathrm{O} 3 \mathrm{w}^{\mathrm{vi}}$ 结晶水则位于对称面两侧, 如图

\section{表 3 化合物 $\mathbf{1}$ 的氢键参数}

\begin{tabular}{|c|c|c|c|c|}
\hline $\mathrm{D}-\mathrm{H} \cdots \mathrm{A}$ & $\mathrm{D}-\mathrm{H}$ & $\mathrm{H} \cdots \mathrm{A}$ & $\mathrm{D} \cdots \mathrm{A}$ & $\mathrm{D}-\mathrm{H} \cdots \mathrm{A}$ \\
\hline O6w-H6wa $\cdots$ O4w & 0.85 & 2.08 & $2.923(6)$ & 174 \\
\hline O6w-H6wb $\cdots$ O4 $4 w^{\text {iv }}$ & 0.85 & 1.94 & $2.790(6)$ & 174 \\
\hline $\mathrm{O} 2 \mathrm{w}-\mathrm{H} 2 \mathrm{wa} \cdots \mathrm{O} 1^{\mathrm{v}}$ & 0.85 & 1.94 & $2.769(3)$ & 164 \\
\hline $\mathrm{O} 3 \mathrm{w}-\mathrm{H} 3 \mathrm{wb}^{\cdots} \cdots \mathrm{O} 5 \mathrm{w}^{\mathrm{vi}}$ & 0.85 & 2.09 & $2.894(4)$ & 157 \\
\hline $\mathrm{O} 3 \mathrm{w}-\mathrm{H} 3 \mathrm{wa} \cdots \mathrm{O} 2$ & 0.85 & 1.88 & $2.725(4)$ & 172 \\
\hline $\mathrm{O} 4 \mathrm{w}-\mathrm{H} 4 \mathrm{wa}^{\cdots} \mathrm{O} 1^{\mathrm{v}}$ & 0.85 & 1.87 & $2.716(4)$ & 180 \\
\hline $\mathrm{O} 4 \mathrm{w}-\mathrm{H} 4 \mathrm{wb} \cdots \mathrm{O} 3 \mathrm{w}$ & 0.85 & 1.91 & $2.759(4)$ & 180 \\
\hline $\mathrm{O} 5 \mathrm{w}-\mathrm{H} 5 \mathrm{wa} \cdots \mathrm{O} 2 \mathrm{w}$ & 0.85 & 1.96 & $2.792(4)$ & 165 \\
\hline $\mathrm{O} 5 \mathrm{w}-\mathrm{H} 5 \mathrm{wb} \cdots \mathrm{O} 3 \mathrm{w}$ & 0.85 & 1.99 & $2.824(4)$ & 165 \\
\hline $\mathrm{O} 1 w-\mathrm{H} 1 w a \cdots \mathrm{O} 2^{\text {vii }}$ & 0.85 & 1.95 & $2.800(4)$ & 180 \\
\hline $\mathrm{O} 1 \mathrm{w}-\mathrm{H} 1 \mathrm{wb} \cdots \mathrm{O} 2^{\text {viii }}$ & 0.85 & 2.14 & $2.992(4)$ & 180 \\
\hline
\end{tabular}

a) Symmetry codes: (iv) $-x+1 / 2,-y+1 / 2,-z+1$; (v) $x, y-1, z$; (vi) $-x, y,-z+1 / 2$; (vii) $-x,-y+1,-z$; (viii) $x,-y+1, z-1 / 2$. 
2(下)所示. 引人注目的是, 当前的水五聚体团簇骨 架采取一种罕见的“三叉星”笼状构型，这也可看成 是三个蝶式水四聚体进一步相互缩合的结构. 在此 笼中, 可以发现两个 $05 \mathrm{w}$ 结晶水分子作为氢键的单 一受体及双重给体, 分别适应了一个 $\mathrm{O} 2 \mathrm{w}$ 及两个 $\mathrm{O} 3 \mathrm{w}$ 结晶水分子. 另一方面, $\mathrm{O} 2 \mathrm{w}$ 水分子仅是作为一 种氢键双重受体、而两个 $\mathrm{O} 3 \mathrm{w}$ 水分子则被发现既 可作为氢键的单一给体又可作为氢键的单一受体. 此五聚体中的 $\mathrm{O} \cdots \mathrm{O}$ 距离处于 $2.792 \sim 2.894 \AA$ 范围内, 平均值为 $2.837 \AA$ (表 3), 实际上接近源于理论计算 的预期值 $2.72 \AA^{[8 c, 31]}$. 所有 $\mathrm{O} \cdots \mathrm{O} \cdots \mathrm{O}$ 角度均落在 $74.4^{\circ} \sim 87.2^{\circ}$ 范围内(表 4), 严重偏离了预期理想的水 四面体几何构型. 就我们所知, 目前的“三叉星”五核 水簇是一例新奇的晶体学范例, 实质上最接近于早 期理论预期所归类的笼式五核水簇模型笼- $6{ }^{[9]}$ (只是 在氢键取向上稍微不同), 迄今为止笼式五核水簇实 例还未被文献描述过 ${ }^{[32]}$, 因而它在水簇化学领域是 前所未有的. 正如理论计算指出的, 笼式构像结构的

表 4 化合物 1 中的水分子之键角和扭角 $\left({ }^{\circ}\right)$

\begin{tabular}{|c|c|}
\hline $\mathrm{O} 3 \mathrm{w}^{\mathrm{vi}} \cdots \mathrm{O} 5 \mathrm{w}^{\mathrm{v}} \cdots \mathrm{O} 2 \mathrm{w}$ & $85.8(1)$ \\
\hline $\mathrm{O} 5 \mathrm{w} \cdots \mathrm{O} 2 \mathrm{w} \cdots \mathrm{O} 5 \mathrm{w}^{\mathrm{vi}}$ & $76.52(9)$ \\
\hline $\mathrm{O} 5 \mathrm{w} \cdots \mathrm{O} 3 \mathrm{w} \cdots \mathrm{O} 5 \mathrm{w}^{\mathrm{vi}}$ & $74.4(1)$ \\
\hline $\mathrm{O} 3 \mathrm{w} \cdots \mathrm{O} 5 \mathrm{w} \cdots \mathrm{O} 3 \mathrm{w}^{\mathrm{vi}}$ & $87.1(1)$ \\
\hline $\mathrm{O} 2 \mathrm{w} \cdots \mathrm{O} 5 \mathrm{w} \cdots \mathrm{O} 3 \mathrm{w}$ & $87.2(1)$ \\
\hline $\mathrm{O} 2 \mathrm{w} \cdots \mathrm{O} 5 \mathrm{w} \cdots \mathrm{O} 3 \mathrm{w}^{\mathrm{vi}}$ & $85.8(1)$ \\
\hline $\mathrm{O} 4 \mathrm{w}^{\cdots} \cdots \mathrm{O} 3 \mathrm{w}^{\cdots} \cdots \mathrm{O} 5 \mathrm{w}^{\mathrm{vi}} \cdots \mathrm{O} 2 \mathrm{w}$ & $-57.1(1)$ \\
\hline $\mathrm{O} 4 \mathrm{w}^{\mathrm{N}} \cdots \mathrm{O} 3 \mathrm{w} \cdots \mathrm{O} 5 \mathrm{w} \cdots \mathrm{O} 2 \mathrm{w}$ & $52.8(2)$ \\
\hline $\mathrm{O} 6 \mathrm{w}^{\mathrm{iv}} \cdots \mathrm{O} 4 \mathrm{w} \cdots \mathrm{O} 3 \mathrm{w} \cdots \mathrm{O} 5 \mathrm{w}^{\mathrm{vi}}$ & $-103.9(3)$ \\
\hline $\mathrm{O} 6 \mathrm{w}^{\mathrm{N}} \cdots \mathrm{O} 4 \mathrm{w} \cdots \mathrm{O} 3 \mathrm{w} \cdots \mathrm{O} 5 \mathrm{w}$ & $98.5(2)$ \\
\hline $\mathrm{O} 6 \mathrm{w}^{\mathrm{iv}} \cdots \mathrm{O} 4 \mathrm{w}^{\mathrm{N}} \cdots \mathrm{O} 3 \mathrm{w} \cdots \mathrm{O} 5 \mathrm{w}$ & $-179.6(3)$ \\
\hline $\mathrm{O} 6 \mathrm{w} \cdots \mathrm{O} 4 \mathrm{w} \cdots \mathrm{O} 3 \mathrm{w} \cdots \mathrm{O} 5 \mathrm{w}^{\mathrm{vi}}$ & $174.2(2)$ \\
\hline
\end{tabular}

${ }^{\text {[a] }}$ Symmetry codes: (iv) $-x+1 / 2,-y+1 / 2,-z+1$; (vi) $-x, y,-z+1 / 2$
五核水簇比起环状构型来说通常比较不稳定, 因为 前者能量估计比低能态种类至少要高出 $1 \mathrm{kcal} / \mathrm{mol}^{[33]}$, 暗示笼式构像结构五核水簇的形成需要获得额外的 稳定化能. 这似乎就是笼式类型在实验观察上缺失 的原因, 甚至更稳定的环状构型五核水簇实例迄今 也仍然十分有限 ${ }^{[9 \mathrm{~d}]}$. 另一方面, 环状 $\left(\mathrm{H}_{2} \mathrm{O}\right)_{4}$ 四核水簇 可以发现是由 $04 \mathrm{w}$ 和 $06 \mathrm{w}$ 结晶水分子及其心对称等 价原子通过氢键作用构成的一种 $D_{2 h}$ 构像结构, 其中, 前者 $\mathrm{O} 4 \mathrm{w}$ 水分子既作为氢键的双重受体又作为氢键 的单一给体，而后者 $\mathrm{O} 6 \mathrm{w}$ 水分子则仅作为一种氢键 的双重给体. 从氢键理论解释的观点来看 ${ }^{[34]}$, 由于较 少的氢键数目效应, $D_{2 h}$ 构像结构对于四核水簇来说 也通常是一种欠稳定的种类,

尽管已经在金属配合物晶体中观察到有限的几 例 ${ }^{[5 \mathrm{e}]}$, 目前的 $D_{2 h}$ 水四聚体仍属于十分稀少的实例. 这些水五聚体及四聚体通过 $\mathrm{O} 4 \mathrm{w} \cdots \mathrm{O} 3 \mathrm{w}$ 氢键作用相 互联结, 产生一种有点扭拧的一维扩展型水带(图 2, 下), 其 $\mathrm{O} \cdots \mathrm{O} \cdots \mathrm{O} \cdots \mathrm{O}$ 扭角处于 $-179.6^{\circ} \sim 174.2^{\circ}$ 范围内 (表 4). 后面将会讨论到, 这种链式水带的连接方式 将有利于水五聚体及四聚体两者结构的稳定性. 此 外，众所周知，在所有水簇之中，一维水结构在控制 多种生物分子的质子流 ${ }^{\left[{ }^{35]}\right.}$ 以及促进生物膜的选择性 水渗透方面扮演着重要角色, 显然, 按照 Infantes 等 ${ }^{[36]}$ 所描述的方法, 根据从剑桥结构数据库 $(\mathrm{CSD})^{[37]}$ 快速检索的结果 ${ }^{[32]}$, 目前的一维扩展型水带代表了 一种全新的一维水结构模式、完全不同于一般那些由 简单水分子 ${ }^{[4 d, 10 d]}$ 无限连接组装或是通过连接一种或 多种形式小环状水簇 ${ }^{[38]}$ 而构成的一维水结构.

如图 3(a)所示, 可以发现化合物 1 中的二维阳离 子及阴离子层沿大致 [001]方向交替堆积成- $\mathrm{ABAB}-$
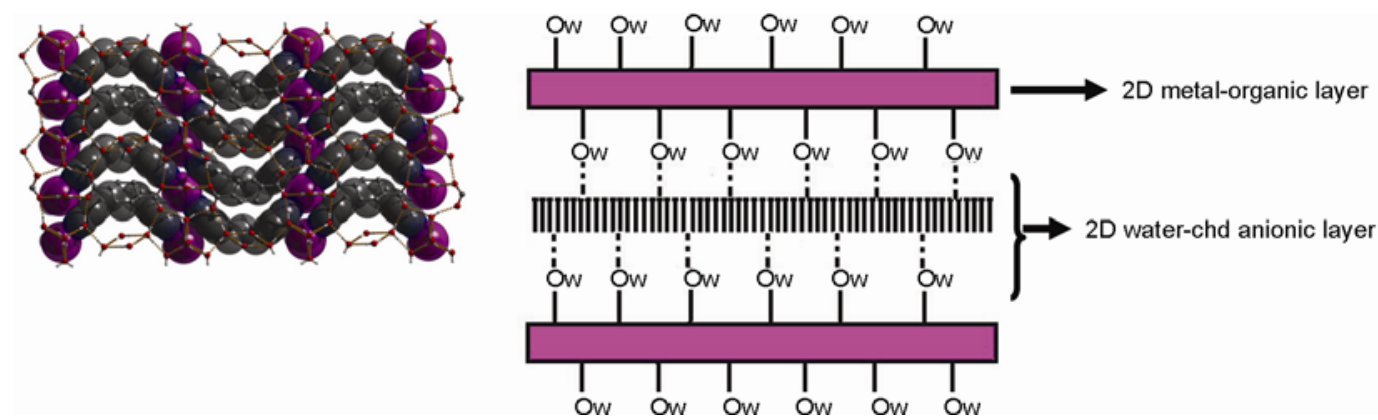

图 3 (a)化合物 $\mathbf{1}$ 中的金属-有机层片(堆积球部分)及水-chd 阴离子网层(球-棍部分)俯视图(沿者 $c$ 轴方向); (b)化合物 $\mathbf{1}$ 的氢 键堆积阵列示意图 
次序结构 $\left(\mathrm{A}\right.$ 和 $\mathrm{B}$ 分别代表 $\left\{\left[\mathrm{Ag}_{2}(\mathrm{bpp})_{2}\left(\mathrm{H}_{2} \mathrm{O}\right)_{2}\right]^{2+}\right\}_{n}$ 阳 离子层和 $\left\{\left[(\mathrm{chd}) \cdot 9 \mathrm{H}_{2} \mathrm{O}\right]^{2-}\right\}_{n}$ 阴离子层), 导致产生一种 二维金属-超分子三文治式网络, 其中那些水-阴离子 层状物填充在金属-有机网络的层间(图 3(b)). 这显得 有点类似于例如蒙㿝石类矿物的行为, 其层间域易 吸水而发生矿物膨胀 ${ }^{[39]}$. 在配位水分子与阴离子层 之间可观察到明显的 $\mathrm{O}-\mathrm{H} \cdots \mathrm{O}$ 氢键作用, 前者提供氢 键给体, 而后者则作为氢键受体(表 3). 显然, 这些广 泛存在的氢键连同静电吸引力是导致驱动这种 $\mathrm{ABAB}$ 阴阳离子层堆垛的原因.

\section{3 水簇的稳定性}

正如上述所指, 笼式五聚体或 $D_{2 h}$ 四聚体水簇均 远非最稳定的种类, 那么关于其组装的一个有趣问 题是: 除了来自主-客体相互作用的几何结构约束贡 献之外, 还有何因素对其稳定性产生贡献? 为了解 其稳定性, 本文采用 Gaussian 03 软件包 ${ }^{[40]}$, 以 6-31+ $\mathrm{G}(\mathrm{d}, \mathrm{P})$ 为基组 ${ }^{[41]}$, 在 B3LYP 精度水准上完成一种基 于密度泛涵理论(DFT)的从头算起计算. 完全基于化 合物 $\mathbf{1}$ 的晶体结构, 没有任何对称性限制的单点能量 计算被用于评估水簇的稳定性. 计算结果表明, 稳定 化能 $\Delta E$ (此处 $\Delta E$ 定义为 $\Delta E=E\left(\mathrm{H}_{2} \mathrm{O}\right)_{n}-n E\left(\mathrm{H}_{2} \mathrm{O}\right), n=$ 水簇聚集数) 对于分立的笼状五聚体及 $D_{2 h}$ 四聚体水 簇分别为 -28.8 及 $-11.3 \mathrm{kcal} / \mathrm{mol}$. 但是, 对于化合物 1
中由上述笼状五聚体及 $D_{2 h}$ 四聚体水簇构成的一个重 复单元 $\left(\mathrm{H}_{2} \mathrm{O}\right)_{9}$ 九核聚集体来说, 发现其计算的 $\Delta E$ 达到 $-59.9 \mathrm{kcal} / \mathrm{mol}$, 远大于两者的算术简单加和 (-40.1 kcal/mol), 这说明由于基于所谓“ $1+1$ 大于 $2^{\text {, [42] }}$ 的“协同效应”有利于降低体系能量贡献，因而五聚 体及四聚体水簇两者的联结有利于增加水链的稳定 性. 图 4 给出了四聚体、五聚体及九聚体水簇的电子 密度和静电势能图, 从中可见其电子离域化特征是 相当明显的, 反映出氢键相互作用对于该新颖水簇 构像结构之形成与稳定化的戏剧性效应.

\section{4 性质与表征}

化合物 $\mathbf{1}$ 的 IR 光谱显示出其 $\mathrm{chd}^{2-}$ 和 bpp 配体的 特征吸收谱带处于 4000 400 $\mathrm{cm}^{-1}$ 区域, 一个归属于 水簇 $\mathrm{O}-\mathrm{H}$ 拉伸振动频率, 中心位于约 $3420 \mathrm{~cm}^{-1}$ 处的 宽吸收谱带在 $100{ }^{\circ} \mathrm{C}$ 真空加热处理 2 小时后消失了, 这肯定是源于晶格中的水分子逃逸所致(图 S2). 文献 报道利用损耗与碎片红外光谱学探究 ${ }^{[43]}$ 气态小分子 水簇 $\left(\mathrm{H}_{2} \mathrm{O}\right)_{n}(n=2 \sim 10)$ 的 $\mathrm{O}-\mathrm{H}$ 拉伸振动, 指出其拉伸 振动分布在 $3720 \sim 2935 \mathrm{~cm}^{-1}$ 较宽的范围内. 作为对 比, 冰的 IR 光谱显示出 $\mathrm{O}-\mathrm{H}$ 拉伸振动吸收谱带发生 在尖锐的 $3220 \mathrm{~cm}^{-1}$ 处, 而相应的液态水拉伸振动也 出现在狭窄的 3490 和 $3280 \mathrm{~cm}^{-1}$ 处 ${ }^{[5 d]}$, 因此, 这个 $3420 \mathrm{~cm}^{-1}$ 处的 $\mathrm{O}-\mathrm{H}$ 拉伸振动显然说明化合物 1 水簇

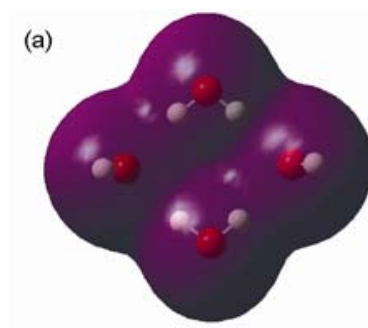

(i)

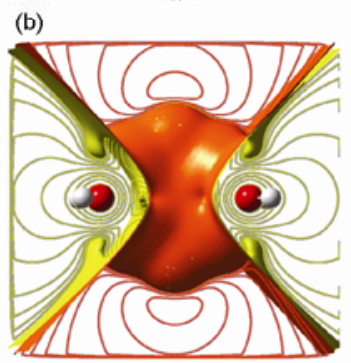

(iv)

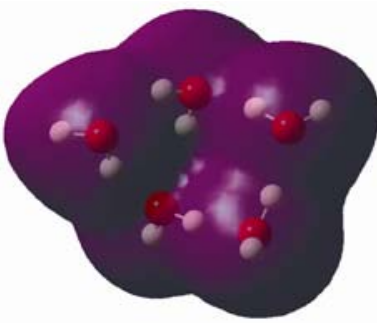

(ii)

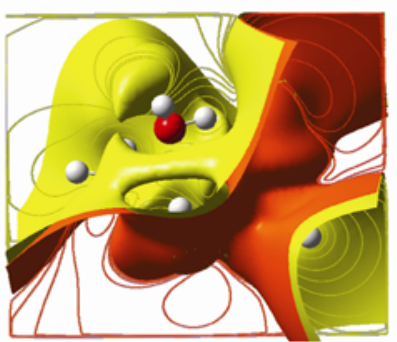

(v)

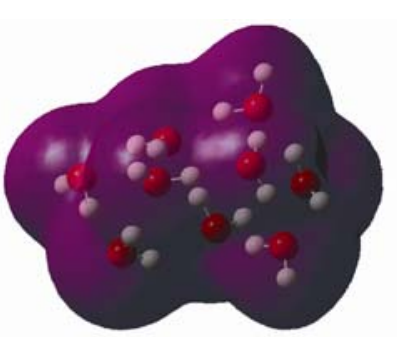

(iii)

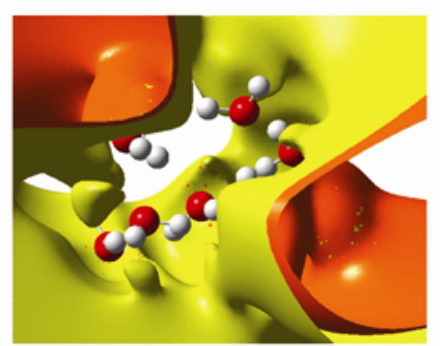

(vi)

图 4 (a)化合物 $\mathbf{1}$ 中水簇的电子密度图: (i)四聚体 $\left(\mathrm{H}_{2} \mathrm{O}\right)_{4}$ 、(ii)五聚体 $\left(\mathrm{H}_{2} \mathrm{O}\right)_{5}$, 及(iii)九聚体 $\left(\mathrm{H}_{2} \mathrm{O}\right)_{9}$ 重复单元; (b)化合物 $\mathbf{1}$ 中水簇 的静电势能图: (iv)四聚体 $\left(\mathrm{H}_{2} \mathrm{O}\right)_{4}$ 、(v)五聚体 $\left(\mathrm{H}_{2} \mathrm{O}\right)_{5}$, 及(vi)九聚体 $\left(\mathrm{H}_{2} \mathrm{O}\right)_{9}$ 重复单元 
行为更接近液态水而非固态冰, 其稍微差别应为小 簇水与体相水之间不同的环境所致 ${ }^{[44]}$, 这也可从该 水链与那些液相水具有相当 $\mathrm{O} \cdots \mathrm{O}$ 距离 $(2.85 \AA)$ 的证 据得以证实. 此外, 化合物 $\mathbf{1}$ 的 1610、1557 及 1420 $\mathrm{cm}^{-1}$ 处吸收峰可归属于其羧基的不对称与对称吸收 振动, 在 1690 1730 $\mathrm{cm}^{-1}$ 区域缺少吸收谱说明 $\mathrm{H}_{2} \mathrm{chd}$ 分子已完全脱除质子 ${ }^{[45]}$, 所有这些均与 $\mathrm{X}$ 射线衍射 技术结构分析结果相吻合.

化合物 1 的热重(TGA) 图谱是在 25 800 ${ }^{\circ} \mathrm{C}$ 范围 采用纯相多晶样品完成的, 如图 S3 所示. TGA 分析 结果表明, 第一个失重发生在 $35 \sim 100{ }^{\circ} \mathrm{C}$ 范围, 大约 失重 $20.0 \%$, 相当于失去所有水分子(计算值 $20.2 \%$ ), 接着发现化合物骨架解体始于约 $200{ }^{\circ} \mathrm{C}$, 先失去有 机配体, 最后发现完全分解完成于 $400{ }^{\circ} \mathrm{C}$.

在当前配位化学领域, 具有可逆吸水-脱水性质 的配合物特别受到关注, 因为这些配合物中客体水 分子的脱除可能会引起单晶到单晶的结构转变 ${ }^{[46]}$. 在上述 TGA 分析基础上, 为了进一步了解客体水的 脱除/重吸稳定性, 图 S4 给出了化合物 $\mathbf{1}$ 样品加热处 理过的粉末 $\mathrm{X}$ 射线衍射图谱(PXRD). 与初始的晶体 相比, $105{ }^{\circ} \mathrm{C}$ 热处理过的失水样品展现出完全不同于 原来的物相, 显然这也许是水分子逸出后, 主体晶格 发生坍塌所致. 而且, 可观察到失水样品及其浸泡于 水中 3 天重新吸水后的谱图虽然强度不同, 但几乎具 有相同衍射峰位置, 表明化合物 $\mathbf{1}$ 的失水是不可逆 的，这意味着化合物 $\mathbf{1}$ 中的水簇更像是一种“胶水”, 起着粘接与稳定整个配合物结构的作用.

由 $\mathrm{d}^{10}$ 组态过渡金属元素与有机芳香性桥联配体 分子构成配合物通常会出现显著的荧光发射性质 ${ }^{[47]}$, 已经观察到某些配位聚合物(CPs)通过混杂金属中心, 特别是 $\mathrm{d}^{10}$ 金属中心, 具有调节有机材料荧光发射波 长能力 ${ }^{[48]}$. 最近, 文献报道含二(吡啶基)配体的 $\mathrm{d}^{10}$ $\mathrm{Ag}(\mathrm{I})$ 配合物也具有荧光发光性质 ${ }^{[49]}$, 因此, 本文对 标题化合物 1, 连同 bpp 配体在室温下的固态荧光发 光性质进行调查, 图 S5 示出化合物 1 及其配体自由 分子的菼光发射谱. 对比孤立的配体 bpp 分子所展现
的苂光性质, 其最大发射峰处于 $445 \mathrm{~nm}$ 处(激发波长 $\lambda_{\mathrm{ex}}=300 \mathrm{~nm}$ ), 大概归属于 $\pi^{*} \rightarrow \mathrm{n}$ 或 $\pi^{*} \rightarrow \pi$ 电子跃迁; 而化合物 1 则观察到强发射出现在 $522 \mathrm{~nm}$ 处 $\left(\lambda_{\mathrm{ex}}=\right.$ $310 \mathrm{~nm}), \mathrm{bpp}$ 与 $\mathrm{Ag}(\mathrm{I})$ 离子配位后红移了约 $77 \mathrm{~nm}$. 一 般地说, 在含有 $\mathrm{N}$ 配体的情况下, 二羧基类配体对所 合成 CPs 的荧光发射没有重要贡献 ${ }^{[50]}$, 因为二羧基 类配体的苂光发射属于 $\pi^{*} \rightarrow \mathrm{n}$ 电子跃迁, 比起 $\mathrm{bpp}$ 的 $\pi^{*} \rightarrow \pi$ 电子跃迁显得十分微弱. 因此, 化合物 1 的苂 光发射应该大概反映出配位 $\mathrm{N}$ 配体之配体内电子跃 迁, 类似的荧光发射性质也在某些配合物与自由含 $\mathrm{N}$ 配体中有所发现 ${ }^{[15]}$. 此外, 化合物 $\mathbf{1}$ 的苂光发射红移 现象主要来自于配体到金属中心的配位作用, 这种 作用通过无辐射衰减途径以及增加了配体刚性, 从 而有效地降低了能量损失 ${ }^{[51]}$.

\section{4 结论}

$\left\{\left[\mathrm{Ag}_{2}(\mathrm{bpp})_{2}\left(\mathrm{H}_{2} \mathrm{O}\right)_{2}\right](\mathrm{chd}) \cdot 9 \mathrm{H}_{2} \mathrm{O}\right\}_{n}$ 固态聚合物中的 “三叉星”笼式五核水聚集体在水簇化学领域中肯定 代表了一种新颖、非凡的水簇类型, 其新颖性部分源 自它不仅属于通过主-客体相互作用提供适宜几何结 构环境所捕获的一种欠稳定物种, 而且在于与另一 欠稳定 $D_{2 h}$ 四核水簇的联接作用形成了一条有趣的一 维扩展型水带. 后者的联接作用显然通过所谓的“协 同效应”对这些欠稳定类水簇的结构稳定性产生贡献, 戏剧性地降低了整个体系的能量. 另外, 晶体学水准 的 $\mathrm{chd}^{2-} \cdot\left(\mathrm{H}_{2} \mathrm{O}\right)_{10}$ 水簇阴离子水化壳为人们了解二羧 基类配体与水相之间相互作用及其分子识别现象提 供了重要的知识.

支持性材料信息：图 S1 S5 包括化合物 $\mathbf{1}$ 的主体聚合 物结构图像、FT-IR 光谱、TGA 热重曲线、说明结晶 水分子不可逆失水性质的粉末 XRD 图谱, 以及苂光 发射光谱; CIF 格式的额外晶体学数据已在剑桥晶体 学数据库中登记为 CCDC 812566.

致谢本工作得到国家自然科学基金(50971063)、福建省自然科学基金(2003F006、2010J01042 和 2011J01047)和教 育部留学回国人员基金项目的资助。作者感谢谢湖均博士(浙江工商大学)、孙迪博士(厦门大学)及 L. Infantes 博士 (Instituto de Química-Física Rocasolano, CSIC, Spain)分别在理论计算、单晶 X 射线衍射测试及 CSD 检 索方面的帮助! 骆耿耿同时也感谢华侨大学科研启动基金(10BS210)的支持. 


\section{参考文献}

1 (a) Eisenberg D, Kauzmann W. The Structure and Properties of Water. Oxford: Oxford University Press, 1969; (b) Haymet ADJ, Dill KA. A simple model of water and the hydrophobic effect. J Am Chem Soc, 1998, 120: 3166-3175; (c) Janiak C, Scharmann TG, Mason SA. Two-dimensional water and ice layers: neutron diffraction studies at 278, 263, and 20 K. J Am Chem Soc, 2002, 124: 14010-14011; (d) Fletcher NH. The Chemical Physics of Ice. Cambridge: Cambridge University Press, 1970

2 (a) Liu K, Brown MG, Carter C, Saykally RJ, Gregory JK, Clary DC. Characterization of a cage form of the water hexamer. Nature, 1996, 381: 501-503; (b) Nauta K, Miller RE. Formation of cyclic water hexamer in liquid helium: the smallest piece of ice. Science, 2000, 287: 293-295; (c) Kim J, Majumdar D, Lee HM, Kim KS. Structures and energetics of the water heptamer: Comparison with the water hexamer and octamer. J Chem Phys, 1999, 110: 9128-9134; (d) Nangia A. ed. Encyclopaedia of Supramolecular Chemistry. New York: Taylor \& Francis, 2007

3 (a) Pan QH, Li JY, Christensen KE, Bonneau C, Ren XY, Shi L, Sun JL, Zou XD, Li GH, Yu JH, Xu RR. A germanate built from a $6^{8} 12^{6}$ cavity co-templated by an $\left(\mathrm{H}_{2} \mathrm{O}\right)_{16}$ cluster and 2-methylpiperazine. Angew Chem Int Ed, 2008, 47: 7868-7871; (b) Bi Y, Liao W, Zhang H, Li D. Assembly of 'discrete' $\left(\mathrm{H}_{2} \mathrm{O}\right)_{16}$ water clusters within a supramolecular compound of calixarene. CrystEngComm, 2009, 11: 1213-1216 (c) Das MC, Bharadwaj PK. Molecular ice with hybrid water-bromide network around a cryptand with a bromide ion included in the cavity to form a host-within-a-host-like structure. Eur J Inorg Chem, 2007, 1229-1232; (d) Mimura M, Matsuo T, Nakashima T, Matsumoto N. Zigzag-chain and cyclic-tetrameric compounds derived by deprotonation of mononuclear copper(II) complexes with N,N'-bis(2-substituted-imidazol-4-ylmethyl-idene)-1,4-diaminobutane (2-Substituent H, Me): Synthesis, characterization, structure, substituent effect, and interconvertibility. Inorg Chem, 1998, 37: 3553-3560; (e) Xie YS, Ni J, Zheng FK, Cui Y, Wang QG, Ng SW, Zhu WH. Tetra- and binuclear complexes of hydroxy-rich ligands: Supramolecular structures, stabilization of unusual water clusters, and magnetic properties. Cryst Growth Des, 2009, 9: 118-126; (f) Custalcean R, Afloroaiei C, Vlassa M, Polverejan M. Formation of extended tapes of cyclic water hexamers in an organic molecular crystal host. Angew Chem Int Ed, 2000, 39: 3094-3096; (g) Lakshminarayanan PS, Suresh E, Ghosh P. A hybrid water-chloride structure with discrete undecameric water moieties self-assembled in a heptaprotonated octaamino cryptand. Angew Chem Int Ed, 2006, 45: 3807-3811; (h) Cheng L, Lin JB, Gong JZ, Sun AP, Ye BH, Chen XM. Encapsulation of water cluster, meso-helical chain and tapes in metal organic frameworks based on double-stranded $\mathrm{Cd}(\mathrm{II})$ helicates and carboxylates. Cryst. Growth Des, 2006, 6: 2739-2746; (i) Xu WZ, Sun JL, Huang ZT, Zheng QY. Molecular encapsulation of a discrete $\left(\mathrm{H}_{2} \mathrm{O}\right)_{32}$ cluster with $S_{6}$ symmetry in an organic crystalline supermolecule. Chem. Commun, 2009, 171-173; (j) Wan YH, Zheng XJ, Wang FQ, Zhou XY, Wang KZ, Jin LP. Water cluster supported architecture of lanthanide coordination polymers with pyrazinetricarboxylic acid. CrystEngComm, 2009, 11: 278-283; (k) Dai FN, He HY, Sun DF. A metal organic nanotube exhibiting reversible adsorption of $\left(\mathrm{H}_{2} \mathrm{O}\right)_{12}$ Cluster. J Am Chem Soc, 2008, 130: 14064-14065; (1) Jin JC, Wang YY, Liu P, Liu RT, Ren C, Shi QZ. An unusual independent 1D metal-organic nanotube with mesohelica structure and 1D-2D Interdigitation. Cryst Growth Des, 2010, 10: 2029-2032; (m) Saha BK, Nangia A. First example of an ice-like water hexamer boat tape structure in a supramolecular organic host. Chem Commun, 2006, 1825-1827; (n) Sun D, Wang DF, Han XG, Zhang N, Huang RB, Zheng LS. Stepwise assembly of two 3d-4d heterometallic coordination polymers based on a hexanuclear silver(I) metalloligand. Chem Commun, 2011, 47: 746-748; (o) Sun D, Wang DF, Zhang N, Huang RB, Zheng LS. Nonamer water cluster encapsulated in a heterometallic supramolecular complex. Cryst Growth Des, 2010, 10: 5031- 5033; (p) Sun D, Xu HR, Yang CF, Wei ZH, Zhang N, Huang RB, Zheng LS. Encapsulated diverse water aggregates in two Ag(I)/4,4'bipyridine/dicarboxylate hosts: 1D water tape and chain. Cryst Growth Des, 2010, 10: 4642-4649; (q) Sun D, Wei ZH, Yang CF, Wang DF, Zhang N, Huang RB, Zheng LS. PH-dependent Ag(I) coordination architectures constructed from 4-cyanopyridine and phthalic acid: from discrete structure to 2D sheet. CrystEngComm, 2011, 13: 1591-1601; (r) Yuan G, Zhu C, Liu Y, Fang Y, Cui Y. Water clusters induced assembly of chiral organic microstructures showing reversible phase transformations and luminescence switching. Chem Commun, 2010, 46: 2307-2309; (s) Sun D, Wang DF, Zhang N, Liu FJ, Hao HJ, Huang RB, Zheng LS. Capture and activation of aerial $\mathrm{CO}_{2}$ by carbamoylation of L-threonine in a $\mathrm{Ag}(\mathrm{I})$ supramolecular framework. Dalton Trans, 2011, 40: 5677-5679

4 (a) Fei Z, Zhao D, Geldbach TJ, Scopelliti R, Dyson PJ, Antonijevic S, Bodenhausen G. A synthetic zwitterionic water channel: characterization in the solid state by X-ray crystallography and nmr spectroscopy. Angew Chem Int Ed, 2005, 44: 5720-5725; (b) Mukherjee A, Saha MK, Nethaji M, Chakravarty AR. Helical supramolecular host with aquapores anchoring alternate molecules of helical water chains. Chem Commun, 2004, 716-717; (c) Natarajan R, Charmant JPH, Orpen AG, Davi AP, Water chains in hydrophobic crystal channels: nanoporous materials as supramolecular analogues of carbon nanotubes. Angew Chem Int Ed, 2010, 49: 5125-5129; (d) Saha BK, Nangia A. Helical water chains in aquapores of organic hexahost: Remarkable halogen-substitution effect on the handedness of water helix. Chem Commun, 2005, 3024-3026; (e) Cui Y, Cao ML, Yang LF, Niu YL, Ye BH. Water nanotubes confined to nanochannels of a (10, 3)-b net constructedby binary building blocks via the $\mathrm{R}_{2}{ }^{2}(9)$ synthon. CrystEngComm, 2008, 10: 1288-1290; (f) Xiong HB, Sun D, Luo GG, 
Huang RB, Dai JC, One 1D T4(0)A(0) water tape embedded in a 1D Cu(II) coordination polymer with 1,3-bis(4-pyridyl) propane. J Mol Struct, 2011, 990: 164-168

5 (a) Shi XF, Song HB, He L, Zhang WQ. Observation of a quasi-one-dimensional water aggregate in a supramolecular organic host. CrystEngComm, 2009, 11: 542-544; (b) Ma BQ, Sun HL, Gao S. Observation of an octameric water cluster containing a book-shaped hexamer in a 4f-3d complex. Chem Commun, 2005, 2336-2338; (c) Tadokoro M, Fukui S, Kitajima T, Nagao Y, Ishimaru S, Kitagawa H, Isobee K, Nakasuji K. Structures and phase transition of multi-layered water nanotube confined to nanochannels. Chem Commun, 2006, 1274-1276; (d) Custelcean R, Afloroaei C, Vlassa M, Polverejan M. Formation of extended tapes of cyclic water hexamers in an organic molecular crystal host. Angew Chem Int Ed, 2000, 39: 3094-3096; (e) Fabelo O, Pasán J, Cañadillas-Delgado L, Delgado FS, Labrador A, Lloret F, Julve M, Ruiz-Pérez C. Well-resolved unusual alternating cyclic water tetramers embedded in a crystal host. CrystEngComm, 2008, 10: 1743-1746

6 (a) Luan XJ, Chu YC, Wang YY, Li DS, Liu P, Shi QZ. Formation of two-dimensional supramolecular water layer containing $\left(\mathrm{H}_{2} \mathrm{O}\right)_{18}$ morphology via dianion templating. Cryst. Growth Des, 2006, 6: 812-814; (b) Oxtoby NS, Blake AJ, Champness NR, Wilson C. Water superstructures within organic arrays; Hydrogen-bonded water sheets, chains and clusters Chem Eur J, 2005, 11: 4643-4654; (c) Zhang JP, Huang XC, Lin YY, Chen XM. Well-resolved, new water morphologies obtained by modification of the hydrophilic/hydro- phobic character and shapes of the supporting layers. Inorg Chem, 2005, 44: 3146-3150; (d) Lakshminarayanan PS, Suresh EI, Ghost P. Formation of an infinite 2D-layered water of $\left(\mathrm{H}_{2} \mathrm{O}\right)_{45}$ cluster in a cryptand-water supramolecular complex. J Am Chem Soc, 2005, 127: 13132-13133; (e) Fei ZF, Geldbach TJ, Zhao DB, Scopelliti R, Dyson PJ. A nearly planar water sheet sandwiched between strontium-imidazolium carboxylate coordination polymers. Inorg Chem, 2005, 44: 5200-5202; (f) Yang AH, Zhang H, Gao HL, Zhang WQ, He L, Cui JZ. Novel water clusters in two complexes of pyridine-2,3,5,6-tetracarboxylate. Cryst Growth Des, 2008, 8: 3354-3359

7 (a) Carballo R, Covelo B, Lodeiro C, Vázquez-López EM. One-dimensional fluorescent stacking structure based on zinc mixed- complex salt encapsulated within an 'ice-like' three-dimensional hydrogen-bonded water network. CrystEngComm, 2005, 7: 294-296; (b) Carballo R, Covelo B, Fernández-Hermida N, García-Martínez E, Lago AB, Vázauez M, Vázauez-López EM. Supramolecular aggregation of hexameric water clusters into a 2D water polymer containing $\left(\mathrm{H}_{2} \mathrm{O}\right)_{18}$ holes. Cryst Growth Des, 2006, 6: 629-631; (c) Li CH, Huang K L, Dou JM, Chi YN, Xu YQ, Shen L, Wang DQ, Hu CW. An interesting six-connected 3D nanowater framework constructed from turbine-type $\left(\mathrm{H}_{2} \mathrm{O}\right)_{18}$ clusters based on a Mn(III) complex. Cryst Growth Des, 2008, 8: 3141-3143

8 (a) Liu K, Btown MG, Cruzan JD, Saykally RJ. Vibration-rotation tunneling spectra of the water pentamer: structure and dynamics. Science, 1996, 271: 62-64; (b) Zabel V, Saenger W, Mason SA. Topography of cyclodextrin inclusion complexes. Part 23. Neutron diffraction study of the hydrogen bonding in .beta.-cyclodextrin undecahydrate at $120 \mathrm{~K}$ : from dynamic flip-flops to static homodromic chains. J Am Chem Soc, 1986, 108: 3664-3673; (c) Zheng JM, Batten S R, Du M. A unique cyanide-bridged three-dimensional (3-D) copper(II)-copper(I) mixed-valence polymer containing 1-D water tapes with cyclic pentamer units. Inorg Chem, 2005, 44: 3371-3372; (d) Ma BQ, Sun HL, Gao S. Cyclic water pentamer in a tape-like structure. Chem Commun, 2004, 2220-2221

9 (a) Ugalde JM, Alkorta I, Elguero J. Water clusters: Towards an understanding based on first principles of their static and dynamic properties. Angew Chem Int Ed, 2000, 39: 717-721; (b) Wales DJ, Walsh TR. Theoretical study of the water pentamer. J Chem Phys, 1996, 105: 6957-6971

10 (a) Barbour LJ, Orr GW, Atwood JL. An intermolecular $\left(\mathrm{H}_{2} \mathrm{O}\right)_{10}$ cluster in a solid-state. Nature, 1998, 393: 671-673; (b) Blanton WB, Gordon-Wylie SW, Clark GR, Jordan KD, Wood JT, Geiser U, Collins TJ. Synthesis and crystallographic characterization of an octameric water complex $\left(\mathrm{H}_{2} \mathrm{O}\right)_{8}$. J Am Chem Soc, 1999, 121: 3551-3552; (c) Barbour LJ, Orr GW, Atwood JL. Characterization of a well resolved supramolecular ice-like $\left(\mathrm{H}_{2} \mathrm{O}\right)_{10}$ cluster in the solid state. Chem Commun, 2000, 859-860; (d) Sreenivasulu B, Vittal JJ. Helix inside a helix: encapsulation of hydrogen-bonded water molecules in a staircase coordination polymer. Angew Chem Int Ed, 2004, 43: 5769-5772

11 (a) Atwood JL, Barbour LJ, Ness TJ, Raston CL, Raston PL. A well-resolved ice-like $\left(\mathrm{H}_{2} \mathrm{O}\right)_{8}$ cluster in an organic supramolecular complex. $J$ Am Chem Soc, 2001, 123: 7192-7193. (b) Pal S, Sankaran NB, Samanta A. Structure of a self-assembled chain of water molecules in a crystal host. Angew Chem Int Ed, 2003, 42: 1741-1743

$12 \mathrm{Ma} \mathrm{BQ}$, Sun HL, Gao S. Formation of two-dimensional supramolecular icelike layer containing $\left(\mathrm{H}_{2} \mathrm{O}\right)_{12}$ rings. Angew Chem Int Ed, 2004, 43: 1374-1376

13 Cheruzel LE, Pometun MS, Cecil MR, Mashuta MS, Wittebort RJ, Buchanan RM. Structures and solid-state dynamics of one-dimen- sional water chains stabilized by imidazole channels. Angew Chem Int Ed, 2003, 42: 5452-5455

14 Raghuraman K, Katti KK, Barbour LJ, Pillarsetty N, Barnes, CL, Katti KV. Characterization of supramolecular $\left(\mathrm{H}_{2} \mathrm{O}\right)_{18}$ water morphology and water-methanol $\left(\mathrm{H}_{2} \mathrm{O}\right)_{15}\left(\mathrm{CH}_{3} \mathrm{OH}\right)_{3}$ clusters in a novel phosphorus functionalized trimeric amino acid host. J Am Chem Soc, 2003, 125: 6955-6961 
15 (a) Luo GG, Xiong HB, Dai JC. Syntheses, structural characterization and properties of $\left\{\left[\mathrm{Cu}(\mathrm{bpp})_{2}\left(\mathrm{H}_{2} \mathrm{O}\right)_{2}\right](\mathrm{tp}) \cdot 7 \mathrm{H}_{2} \mathrm{O}\right\}$ and $\left\{\left[\mathrm{Cu}(\mathrm{bpp})_{2}\left(\mathrm{H}_{2} \mathrm{O}\right)\right](\mathrm{ip}) \cdot 7 \mathrm{H}_{2} \mathrm{O}\right\}$ complexes. New examples of organic anionic template-effect on induced assembly of water clusters. Cryst Growth Des, 2011, 11: 507-515; (b) Wang XL, Qin C, Wang EB. Polythreading of infinite 1D chains into different structural motifs: Two poly(pseudo-rotaxane) architectures constructed by concomitant coordinative and hydrogen bonds. Cryst Growth Des, 2006, 6: 439-443; (c) Luo GG, Xiong HB, Sun D, Wu DL, Huang RB, Dai JC. A discrete spirocyclic $\left(\mathrm{H}_{2} \mathrm{O}\right)_{9}$ cluster and 1D novel water chain with tetrameric and octameric clusters in cationic hosts. Cryst Growth Des, 2011, 11: 1948-1956

16 Chen B, Park JM, Iavnov I, Tabacchi G, Klein ML, Parrinello M. First-principles study of aqueous hydroxide solutions. J Am Chem Soc, 2002, 124: 8534-8535

17 (a) Achatz U, Fox BS, Beyer MK, Bondybey VE. Hypoiodous acid as guest molecule in protonated water clusters: a combined FT-ICR/ DFT study of $\mathrm{I}\left(\mathrm{H}_{2} \mathrm{O}\right)_{n}$. J Am Chem Soc, 2001, 123: 6151-6160; (b) Cho SJ, Hwang HS, Park JM, Oh KS, Kim KS. Starands vs ketonands: ab initio study. J Am Chem Soc, 1996, 118: 485-486; (c) Kim KS, Lee JY, Lee SJ, Ha TS, Kim DH. On binding forces between aromatic ring and quaternary ammonium compound. J Am Chem Soc, 1994, 116: 7399-7400

18 (a) Minofar B, Vrbka L, Mucha M, Jungwirth P, Yang X, Wang XB, Fu YJ, Wang LS. Interior and interfacial aqueous solvation of benzene dicarboxylate dianions and their methylated analogues: A combined molecular dynamics and photoelectron spectroscopy study. $J$ Phys Chem A, 2005, 109: 5042-5049; (b) Minofar B, Mucha M, Jungwirth P, Yang X, Fu Y J, Wang XB, Wang LS. Bulk versus interfacial aqueous solvation of dicarboxylate dianions. J Am Chem Soc, 2004, 126: 11691-11698

19 Higashi T. ABSCOR, Empirical absorption correction based on fourier series approximation, Rigaku Corporation, Tokyo, 1995

20 Sheldrick GM. SHELXS-97, Program for X-ray crystal structure determination, University of Gottingen, Germany, 1997

21 Sheldrick GM. SHELXL-97, Program for X-ray crystal structure refinement, University of Gottingen, Germany, 1997.

22 Spek AL. Implemented as the PLATON procedure, a multipurpose crystallographic tool, Utrecht University, Ultrecht, The Netherlands, 1998

23 Brandenburg K. DIAMOND, Version 3.1f, Crystal Impact GbR, Bonn, Germany

24 Materials Studio Program, Version 4.3, Accelrys, San Diego, CA, 2008

25 (a) Sun DF, Cao R, Bi WH, Hong MC, Chang YL. Syntheses and characterizations of a series of silver-carboxylate polymers. Inorg Chim Acta, 2004, 357: 991-1001; (b) Smith G, Reddy AN, Byriel KA, Kennard CHL. Preparation and crystal structures of the silver(I) carboxylates $\left[\mathrm{Ag}_{2}\left\{\mathrm{C}_{6} \mathrm{H}_{4}\left(\mathrm{CO}_{2}\right)_{2}\right\}\left(\mathrm{NH}_{3}\right)_{2}\right],\left[\mathrm{NH}_{4}\right]\left[\mathrm{Ag}_{5}\left\{\mathrm{C}_{6} \mathrm{H}_{3}\left(\mathrm{CO}_{2}\right)_{3}\right\}_{2}-\left(\mathrm{NH}_{3}\right)_{2}\left(\mathrm{H}_{2} \mathrm{O}\right)_{2}\right] \cdot \mathrm{H}_{2} \mathrm{O}$ and $\left[\mathrm{NH}_{4}\right]\left[\mathrm{Ag}\left\{\mathrm{C}_{4} \mathrm{H}_{2} \mathrm{~N}_{2}\left(\mathrm{CO}_{2}\right)_{2}\right\}\right]$. JCS Dalton Trans, $1995,3565-3570$

26 Hao HQ, Liu WT, Tan W, Lin ZJ, Tong ML. Enantiopure and racemic sandwich-like networks with dehydration, readsorption, and selective guest-exchange phase transformations. Cryst Growth Des, 2009, 9: 457-465

27 Carlucci L, Ciani G, Proserpio DM, Rizzato S. New polymeric networks from the self-assembly of silver(I) salts and the flexible ligand 1,3-bis(4-pyridyl)propane (bpp). A systematic investigation of the effects of the counterions and a survey of the coordination polymers based on bpp. CrystEngComm, 2002, 4: 121-129

28 (a) Mascal M, Kerdelhue JL, Blake AJ, Cooke PA, Mortimer RJ, Teat SJ. On the nature of arene $\eta^{6}$ interactions in the solid state and the use of cylindrophanes as ligands for sandwich complexation of metals with longer-range interactions with the benzene ring. Eur J Inorg Chem, 2000, 485-490; (b) Zheng XF, Zhu LG. Influence of different N-donor ligands on the supramolecular architectures and abundant weak interactions of silver 2-sulfobenzoate polymers. Cryst Growth Des, 2009, 9: 4407-4417

29 (a) Jin JC, Wang YY, Zhang WH, Lermontov AS, Lermontova EK, Shi QZ. New types of di-, tetra-, hexa- and octanuclear Ag(I) complexes containing 1,3-adamantanedicarboxylic acid. Dalton Trans, 2009, 10181-10191; (b) Pan L, Woodlock EB, Wang XT, Lam KC, Rheingold AL. Novel silver(I)-organic coordination polymers: conversion of extended structures in the solid state as driven by argentophilic interactions. Chem Commun, 2001, 1762-1763; (c) Carlucci L, Ciani G, Gudenberg DWV, Proserpio DM. Self-assembly of infinite double helical and tubular coordination polymers from $\mathrm{Ag}\left(\mathrm{CF}_{3} \mathrm{SO}_{3}\right)$ and 1,3-bis(4-pyridyl)propane. Inorg Chem, 1997, 36: 3812-3813

30 Seinfeld JH, Pankow JF. Organic atmospheric particulate material. Annu Rev Phys Chem, 2003, 54: 121-140

31 Naskar JP, Drew MGB, Hulme A, Tocher DA, Datta D. Occurrence of ribbons of cyclic water pentamers in a metallo-organic framework formed by spontaneous fixation of $\mathrm{CO}_{2}$. CrysEngComm, 2005, 7: 67-70

32 The results of CSD search show that there are approximately 24 structures probably contain the water motif of cage-like $\left(\mathrm{H}_{2} \mathrm{O}\right)_{5}$ cluster, i.e. CEYMIC, COENCL, COTENC, CRTENC, DOGRIA, GIZLIL, LEZJOQ, LUNNIS, NUVLEW, PERRAF02, POLDOK, POLDUQ, POZCUC, QAQLAV, QAZJIK, QUFGEE, RUPGOZ, TEGZUA, TOHPIQ, TOHPOW, UDUKEK, WABKIU, WEVRUK and NUPDOS01, of which only 9 structures (COENCL, GIZLIL, LUNNIS, NUPDOS01, POZCUC, QAQLAV, RUPGOZ, TOHPIQ and TOHPOW) are suspected to have the analogous "star" cage water motif in the further inspection. However, all of these suspected "star" cage water motifs 
gave the larger U values or badly ellipsoids without detailed hydrogen sites and their water structures did not have been described and discussed.

33 Burke LA, Jensen JO, Jensen JL, Krishanan PN. Theoretical study of water clusters. I. Pentamer. Chem Phys Lett, 1993, 206: 293-296

34 Masella M, Gresh N, Flament JP. A theoretical study of nonadditive effects in four water tetramers. JCS Faraday Trans, 1998, 94: 27452753

35 (a) Sainz G, Carrell CJ, Ponamerev MV, Soriano GM, Cramer WA, Smith JL. Interruption of the internal water chain of cytochrome f impairs photosynthetic function. Biochemistry, 2000, 39: 9164-9173; (b) Jude KM, Wright SK, Tu C, Silverman DN, Viola RE, Chrisianson DW. Crystal structure of F65A/Y131C-methlimidazole carbonic anhydrase V reveals architectural features of an engineered proton shuttle. Biochemistry, 2002, 41: 2485-2491

36 (a) Ye BH, Sun AP, Wu TF, Weng YQ, Chen XM. Tapes of cyclic water tetramers in the double-helical complex $\left[\mathrm{Cd}_{2}\left(\mathrm{bpa}_{2} \mathrm{Cl}_{4}\right] \cdot 6 \mathrm{H}_{2} \mathrm{O}\right.$. Eur J Inorg Chem, 2005, 1230-1234; (b) Ma BQ, Sun HL, Gao S. Vertex-sharing water tape consisting of cyclic hexamers. Eur J Inorg Chem, 2005, 3902-3906; (c) Jin Y, Che Y, Batten SR, Chen P, Zheng J. Unusual T4(1) water chain stabilized in the one-dimensional chains of a copper(II) coordination polymer. Eur J Inorg Chem, 2007, 1925-1935

37 Allen FH. The cambridge structural database: A quarter of a million crystal structures and rising. Acta Crystallogr Sect B: Struct Sci, 2002, 58: $380-388$

38 (a) Infantes L, Motherwell S. Water clusters in organic molecular crystals. CrystEngComm, 2002, 4: 454-461; (b) Mascal M, Infantes L, Chisholm J. Water oligomers in crystal hydrates-what's news and what isn't? Angew Chem Int Ed, 2006, 45: 32-36

39 Becke AD. Density-functional thermochemistry. III. The role of exact exchange. J Chem Phys, 1993, 98: 5648-5652

40 Frisch MJ, Trucks GW, Schlegel HB, Scuseria GE, Robb MA, Cheeseman JR, Montgomery JA, Vreven T, Kudin KN, Burant JC, Millam JM, Iyengar SS, Tomasi J, Barone V, Mennucci B, Cossi M, Scalmani G, Rega N, Petersson GA, Nakatsuji H, Hada M, Ehara M, Toyota K, Fukuda R, Hasegawa J, Ishida M, Nakajima T, Honda Y, Kitao O, Nakai H, Klene M, Li X, Knox JE, Hratchian HP, Cross JB, Adamo C, Jaramillo J, Gomperts R, Stratmann RE, Yazyev O, Austin AJ, Cammi R, Pomelli C, Ochterski JW, Ayala PY, Morokuma K, Voth GA, Salvador P, Dannenberg JJ, Zakrzewski VG, Dapprich S, Daniels AD, Strain MC, Farkas O, Malick DK, Rabuck AD, Raghavachari K, Foresman JB, Oritiz JV, Cui Q, Baboul AG, Clifford S, Cioslowski J, Stefanov BB, Liu G, Liashenko A, Piskorz P, Komaromi I, Martin RL, Fox DJ, keith T, Al-Laham MA, Peng CY, Nanayakkara A, Challacombe M, Gill PMW, Johnson B, Chen W, Wong MW, Gonzalez C, Pople JA. Gaussian 03, revision B.05; Gaussian, Inc., Pittsburgh, PA, 2003

41 de Pablo L, Chávez ML, Sum AK, de Pablo JJ. Monte Carlo molecular simulation of the hydration of Na-montmorillonite at reservoir conditions. J Chem Phys, 2004, 120: 939-947

42 Prins LJ, Reinhoudt DN, Timmerman P. Noncovalent synthesis using hydrogen bonding. Angew Chem Int Ed, 2001, 40: 2382-2426

43 Buck U, Huisken F. Infrared spectroscopy of size-selected water and methanol clusters. Chem Rev, 2000, 100: 3863-3890

44 Ghosh SK, Bharadwaj PK. Structure of a discrete hexadecameric water cluster in a metal-organic framework structure. Inorg Chem, 2004, 43: 6887-6689

45 (a) Bellamy LJ. The infrared spectra of complex molecules, Wiley: New York, 1958. (b) Zhang ML, Li DS, Wang JJ, Fu F, Du M, Zou K, Gao XM. Structural diversity and properties of $\mathrm{Zn}^{\mathrm{II}}$ and $\mathrm{Cd}^{\mathrm{II}}$ complexes with a flexible dicarboxylate building block 1,3-phenylenediacetate and various heterocyclic co-ligands. Dalton Trans. 2009, 5355-5364

46 (a) Li CP, Du M. Role of solvents in coordination supramolecular systems. Chem Commun, 2011, 47: 5958-5972; (b) Chen XD, Zhao XH, Chen M, Du M. A 3D copper(II) coordination framework showing different kinetic and thermodynamic crystal transformations through removal of guest water cubes. Chem Eur J, 2009, 15: 12974-12977; (c) Zhang XM, Fang RQ, Wu HS. Extended water tapes of cyclic hexamers encapsulated in the channels of a metal phosphonocarboxylate network. Cryst Growth \& Des, 2005, 5: 1335-1337

47 (a) Dai JC, Fu ZY, Wu XT. Supramolecular Coordination Polymers. in Encyclopedia of Nanoscience and Nanotechnology. Nalwa HS, ed. American Scientific Publishers, 2004, 10: 247-266; (b) Dai JC, Wu XT, Fu ZY, Cui CP, Hu SM, Du WX, Wu LM, Zhang HH, Sun RQ. Synthesis, structure, and fluorescence of the novel cadmium(II)-trimesate coordination polymers with different coordination architectures. Inorg Chem, 2002, 41: 1391-1396; (c) Dai JC, Wu XT, Fu ZY, Hu SM, Du WX, Cui CP, Wu LM, Zhang HH, Sun RQ. A novel ribbon-candy-like supramolecular architecture of cadmium-terephthalate polymer with giant rhombic channels: two-fold interpenetration of the 3D 8²10-a net. Chem Commun, 2002, 12-13; (d) Yang GD, Dai JC, Lian YX, Wu WS, Lin JM, Hu SM, Sheng TL, Fu ZY, Wu XT. $\left[\mathrm{Ph}_{3} \mathrm{PCH}_{2} \mathrm{Ph}\right]_{2}\left[\mathrm{Zn}_{3}(\mathrm{tp})_{3} \mathrm{Cl}_{2}\right]$ and $\mathrm{Ni}_{3}(\mathrm{tma})_{2}\left(\mathrm{H}_{2} \mathrm{O}\right)_{8}$ : Two unusual claylike frameworks of metal-polycarboxylate coordination polymers $(\mathrm{tp}=$ terephthalate, tma = trimesate). Inorg Chem, 2007, 46: 7910-7916; (e) Liu L, Huang SP, Yang GD, Zhang H, Wang XL, Fu ZY, Dai JC. $\mathrm{Zn}[\mathrm{Htma}][\mathrm{ddm}]:$ An interesting three-dimensional chiral nonlinear optical-active zinc-trimesate framework. Cryst Growth Des, 2010, 10: 930-936; (f) Lian YX, Yang GD, Fu ZY, Wang XL, Liu L, Dai JC. Cationic induced assembly of two 2D zinc-tereph- thalate polymeric 
networks. Inorg Chim Acta, 2009, 362: 3901-3909; (g) Dai JC, Wu XT, Hu SM, Fu ZY, Zhang JJ, Du WX, Zhang HH, Sun RQ. Crystal engineering of the coordination architecture of metal polycarboxylate complexes by hydrothermal synthesis: assembly and characterization of four novel cadmium polycarboxylate coordination polymers based on mixed ligands. Eur J Inorg Chem, 2004, 2096-2106; (h) Dai JC, Hu SM, Wu XT, Fu ZY, Du WX, Zhang HH, Sun RQ. A novel 2D bilayer architecture generated via $\pi-\pi$ interactions and host-guest molecular recognition: Assembly and structure of $\left\{\left[\mathrm{Cd}(\mathrm{Htma})(\mathrm{bpy})\left(\mathrm{H}_{2} \mathrm{O}\right)\right] \cdot\left(\mathrm{H}_{2} \mathrm{tp}\right)_{0.5} \cdot 2 \mathrm{H}_{2} \mathrm{O}\right\}_{n}$ polymer $\left(\mathrm{tma}=\right.$ trimesate, bpy $=4,4^{\prime}-$ bipyridine, tp = terephthalate). New J Chem, 2003, 23: 914-918

48 (a) Su Z, Xu J, Fan J, Liu DJ, Chu Q, Chen MS, Chen SS, Liu GX, Wang XF, Sun WY. Synthesis, crystal structure, and photoluminescence of coordination polymers with mixed ligands and diverse topologies. Cryst Growth Des, 2009, 9: 2801-2811; (b) Chen SS, Fan J, Okamura TA, Chen MS, Su Z, Sun WY, Ueyama N. Synthesis, crystal structure, and photoluminescence of a series of zinc(II) coordination polymers with 1,4-di(1h-imidazol-4-yl)benzene and varied carboxylate ligands. Cryst Growth Des, 2010, 10: 812-822

49 (a) Chi YN, Huang KL, Cui FY, Xu YQ, Hu CW. Structural diversity of silver(I) 4,6-dipyridyl-2-aminopyrimidine complexes: effect of counteranions and ligand isomerism. Inorg Chem, 2006, 45: 10605- 10612; (b) Fei BL, Sun WY, Okamura T, Tang WX, Ueyama N. Synthesis and crystal structure of a luminescent infinite 2D brick-wall network with two- and three-coordinate silver(I) atoms and ligand-unsupported silver-silver interactions. New J Chem, 2001, 25: 210-212

50 (a) Chen WJ, Wang Y, Chen C, Yue Q, Yuan HM, Chen JS, Wang SN. Photoluminescent metal-organic polymer constructed from trimetallic clusters and mixed carboxylates. Inorg Chem, 2003, 42: 944-946; (b) Shi X, Zhu GS, Wang XH, Li GH, Fang QR, Zhao XJ, Wu G, Tian G, Xue M, Wang RW, Qiu SL. Polymeric frameworks constructed from a metal organic coordination compound, in 1-D and 2-D systems: synthesis, crystal structures, and fluorescent properties. Cryst Growth Des, 2005, 5: 341-346

51 Wu G, Wang XF, Okamura TA, Sun WY, Ueyama N. Syntheses, structures, and photoluminescence properties of metal(II) halide complexes with pyridine-containing flexible tripodal ligands. Inorg Chem, 2006, 45: 8523-8532; (b) Zhu HF, Fan J, Okamura TA, Sun WY, Ueyama N. Syntheses and structures of zinc(II), silver(I), copper(II), and cobalt(II) complexes with imidazole-containing ligand: 1-(1-imidazolyl)-4-(imidazol-1-ylmethyl) benzene. Cryst Growth Des, 2005, 5: 289-294

\title{
Observation of the novel "three-pointed star" cage-like $\left(\mathrm{H}_{2} \mathrm{O}\right)_{5}$ cluster in a polymeric solid $\left\{\left[\mathrm{Ag}_{2}(\mathrm{bpp})_{2}\left(\mathrm{H}_{2} \mathrm{O}\right)_{2}\right](\mathrm{chd}) \cdot 9 \mathrm{H}_{2} \mathrm{O}\right\}_{n}$
}

\author{
LUO GengGeng ${ }^{1}, \mathrm{XIONG}$ HongBo $^{1}$, FU ZhiYong ${ }^{2}$, DAI JingCao ${ }^{1 *}$ \\ 1 Institute of Materials Physical Chemistry, Huaqiao University, Xiamen 361021, China \\ 2 School of Chemistry and Chemical Engineering, South China University of Technology, Guangzhou 510640, China \\ *Corresponding author (email: djc@hqu.edu.cn)
}

\begin{abstract}
Coordination polymeric solid, $\left\{\left[\mathrm{Ag}_{2}(\mathrm{bpp})_{2}\left(\mathrm{H}_{2} \mathrm{O}\right)_{2}\right](\mathrm{chd}) \cdot 9 \mathrm{H}_{2} \mathrm{O}\right\}_{n}(\mathbf{1})\left(\mathrm{bpp}=1\right.$,3-bis(4-pyridyl) propane, $\mathrm{H}_{2}$ chd $=$ 1,4-cyclo- hexanedicarboxylic acid), has been obtained by the solution phase ultrasonic synthesis techniques. The structure established through X-ray structural analysis shows that the compound traps an interesting 1D water tape built by the alternating "three-pointed star" cage-like pentameric and $D_{2 h}$ tetrameric clusters $(C 2 / c, Z=4 ; a=30.37(2) \AA, b=9.271(5)$ $\AA$, and $c=18.89(1) \AA ; \beta=128.47^{\circ} ; V=4164(4) \AA^{3}$ ). The novelty of the present complex is the rarely crystallographic example of the cage-shaped water pentamer, which is usually ascribed to a less-stable species. A first-principle density functional theory (DFT) calculation demonstrates that the interconnectivity between cage-like pentamers and $D_{2 h}$ tetramers is beneficial for contribution to the structural stabilization of these less-stable water cluster species.
\end{abstract}

Keywords: water cluster, water pentamer, cage-like, host, DFT 